\title{
Commentary: Case Report: Abdominal Lymph Node Metastases of Parathyroid Carcinoma: Diagnostic Workup, Molecular Diagnosis, and Clinical Management
}

OPEN ACCESS

Edited by:

Piero Ferolla

Umbria Regional Cancer Network (Rete Oncologica Regionale Umbria,

Perugia), Italy

Reviewed by:

Marco Gallo,

Azienda Ospedaliera Nazionale SS. Antonio e Biagio e Cesare Arrigo, Italy Jessica Costa-Guda, University of Connecticut

United States

${ }^{*}$ Correspondence:

Giuseppe Fanciulli gfanciu@uniss.it

${ }^{\dagger}$ These authors share first authorship

${ }^{\ddagger}$ Membership of NIKE Group is provided in the Acknowledgments

Specialty section: This article was submitted to Cancer Endocrinology, a section of the journal Frontiers in Endocrinology

Received: 26 April 2021 Accepted: 17 May 2021 Published: 18 June 2021

Citation: Fanciulli G, Di Molfetta S, Dotto A, Florio T, Feola T, Colao A, Faggiano A and NIKE Group (2021) Commentary: Case Report: Abdominal Lymph Node Metastases of Parathyroid

Carcinoma: Diagnostic Workup, Molecular Diagnosis, and Clinical Management.

Front. Endocrinol. 12:700806. doi: 10.3389/fendo.2021.700806

\begin{abstract}
Giuseppe Fanciulli ${ }^{1 *}$, Sergio Di Molfetta ${ }^{2 \dagger}$, Andrea Dotto ${ }^{3,4}$, Tullio Florio ${ }^{4,5}$, Tiziana Feola ${ }^{6,7}$, Annamaria Colao ${ }^{8}$, Antongiulio Faggiano ${ }^{9}$ and NIKE Group ${ }^{\ddagger}$

${ }^{1}$ NET Unit, Department of Medical, Surgical and Experimental Sciences, University of Sassari-Endocrinology Unit, Sassari, Italy, ${ }^{2}$ Section of Internal Medicine, Endocrinology, Andrology and Metabolic Diseases, Department of Emergency and Organ Transplantation, University of Bari Aldo Moro, Bari, Italy, ${ }^{3}$ Endocrinology Unit, IRCCS Ospedale Policlinico San Martino, Genova, Italy, ${ }^{4}$ Department of Internal Medicine, University of Genova, Genova, Italy, ${ }^{5}$ IRCCS Ospedale Policlinico San Martino, Genova, Italy, ${ }^{6}$ Department of Experimental Medicine, "Sapienza" University of Rome, Rome, Italy,

7 Neuroendocrinology, Neuromed Institute, IRCCS, Pozzilli, Italy, ${ }^{8}$ Endocrinology Unit, Department of Clinical Medicine and Surgery, University Federico II, Naples, Italy, ${ }^{9}$ Endocrinology Unit, Department of Clinical and Molecular Medicine, Sant'Andrea Hospital, Sapienza University of Rome, Rome, Italy
\end{abstract}

Keywords: parathyroid carcinoma, immune checkpoint inhibitors, ipilimumab, nivolumab, pembrolizumab

\section{A Commentary on}

Case Report: Abdominal Lymph Node Metastases of Parathyroid Carcinoma: Diagnostic Workup, Molecular Diagnosis, and Clinical Management

By Lenschow C, Fuss CT, Kircher S, Buck A, Kickuth R, Reibetanz J, Wiegering A, Stenzinger A, Hübschmann D, Germer CT, Fassnacht M, Fröhling S, Schlegel N and Kroiss M (2021). Front. Endocrinol. 12:643328. doi: 10.3389/fendo.2021.643328

\section{INTRODUCTION}

In the issue of March 2021, Lenschow et al. reported the case of a 46-year-old woman with recurrent, programmed death-ligand-1 (PD-L1) negative, tumor mutational burden (TMB)-high parathyroid carcinoma (PC), who showed stable disease as her best response on imaging, and a three-fold drop in PTH after treatment with intravenous pembrolizumab (1).

Parathyroid carcinoma is a rare neuroendocrine tumour, accounting for $<1 \%$ of all cases of primary hyperparathyroidism (2). While surgery represents the mainstay of treatment for both the primary tumour and metastasis, patients no longer amenable to surgical resection often receive unsatisfactory systemic therapies including cinacalcet, adjuvant radiotherapy, and alkylating agents (3).

In recent years, modulation of immune checkpoint proteins expression has been accounted as a prominent mechanism for tumour immune evasion and survival, thus paving the way for new therapeutic approaches (4). Of note, monoclonal antibodies targeting the programmed cell death-1 (PD-1)/PD-L1 and/or the cytotoxic T lymphocyte antigen-4 (CTLA-4)-B7 pathway, hereinafter collectively referred as immune checkpoints inhibitors (ICIs), have shown both clinical effectiveness and a favorable safety profile in patients with advanced solid tumours, and have been included in the treatment repertoire of several malignancies (5).

Given the remarkable results obtained by Lenschow et al. with the anti-PD-1 agent pembrolizumab in the above-mentioned case, we performed an extensive search for possible further relevant data 
sources, including a) full published articles in international online databases (PubMed, Web of Science, Scopus, and Embase); b) preliminary reports in selected international meeting abstract repositories (American Society of Clinical Oncology, ASCO; European Neuroendocrine Tumor Society, ENET; European Society for Medical Oncology, ESMO); c) registered clinical trials in the U.S. National Institutes of Health registry of clinical trials (http://clinicaltrials.gov) and in any primary register of the WHO International Clinical Trials Registry Platform (ICTRP).

\section{FINDINGS}

a. The search for full-published articles revealed 263 papers, two of which were pertinent to our aim (one of these being the article by Lenschow et al.). In 2020, Park et al. (6) reported the case of a 65year-old man with recurrent hyperparathyroidism and histologically confirmed metastatic PC, who showed objective response as defined by the Response Evaluation Criteria in Solid Tumors version 1.1 (RECIST v1.1) (7) after pembrolizumab administration. The tumour was assessed as PD-L1 negative by immunohistochemistry. Mutations in the MSH2 and MSH6 DNA mismatch repair genes, possibly resulting in high replication error at microsatellite loci, were found in tumour samples through comprehensive gene profiling analysis. Therefore, the patient was deemed eligible for treatment with pembrolizumab. Immune blockade of PD-1 resulted in sustained reduction of pulmonary metastatic tumour burden, with concurrent normalization of both calcium and parathyroid hormone levels.

b. We found no preliminary reports in the above-mentioned international meeting abstract repositories.

c. The search in clinical trial registers revealed two active trials, one of which fully matched our aim. NCT02834013 (DART: Dual Anti-CTLA-4 and Anti-PD-1 Blockade in Rare Tumors) is a Phase 2 study evaluating the effects of nivolumab plus ipilimumab (arm I) versus nivolumab alone (arm II) in patients with rare solid tumours (94 listed histotypes, including PC). The primary outcome is the RECIST v1.1 objective response-rate. Major secondary outcomes include incidence of adverse events, best response, clinical benefit rate, overall survival, and progression free survival. The present study status is "Recruiting." However, according to a very recent update of the protocol, accrual of parathyroid gland tumours has been closed.

\section{DISCUSSION}

To date, very limited evidence is available about the efficacy of ICIs in patients with PC. With this regard, some points should be taken into account.

PD-L1 expression in pre-treatment tumour samples has been proposed as a marker for clinical response to anti-PD-1/PD-L1 immunotherapy in patients with advanced malignancies, including melanoma, non-small cell lung cancer, kidney cancer, colorectal cancer, and castration-resistant prostate cancer $(8,9)$. Notably, immunohistochemistry-assessed PD-L1 expression was found in $4 / 18$ patients $(22.2 \%)$ with histologically confirmed PC (10), thus suggesting that immune checkpoint blockade may have a rationale in the treatment of this type of tumours. While PD-L1-overexpressing tumours tend to have more intense responses, experience with melanoma suggests that PD-1/PD-L1 blockade may be beneficial also in patients with low PD-L1 expression (11-13), therefore a negative PD-L1 status assessment should not definitively preclude the use of ICIs.

There is growing evidence that the TMB can also predict response to ICIs, with the high TMB-patients exhibiting a higher response rate to anti-PD-1/PD-L1 agents possibly due to increased neo-antigen load and $\mathrm{T}$ cell infiltration in the tumour microenvironment $(14,15)$. In a cohort of 16 patients with PC, Kang et al. have recently found three cases with high (>20 m/Mb) TMB through comprehensive genomic profiling (16). Given the higher response rate observed in the high TMBpatients, assessment of mismatch repair status and/or exome sequencing in tumour samples may help identify those patients possibly benefiting the most from administration of anti-PD-1/ PD-L1 agents, in this way enabling a more personalized approach to treatment. The above-mentioned cases of PD-L1 negative, TMB-high tumours benefiting from pembrolizumab therapy further support this approach.

Moreover, PD-1 and CTLA-4 are acknowledged to exert nonredundant immunosuppressive effects (17). As there is robust evidence supporting a greater efficacy of the combined PD-1/ CTLA-4 blockade over the two monotherapies in advanced solid cancer (18), the possible inclusion of patients with PC in the NCT02834013 trial is giving rise to great expectations. Of note, ICI two-drug combination therapy is under evaluation also in patients with other aggressive endocrine tumours (19-22).

As a further reason of interest, hypocalcemia due to immunerelated hypoparathyroidism has been reported as a rare complication following anti-PD-1 therapy initiation in patients with non-parathyroid tumours $(23,24)$. As a result, mitigation of hypercalcemia could be hypothesized as a beneficial adjunctive effect of anti-PD-1 agents in patients with PC, irrespective of their imaging response assessment.

In summary, currently available treatments for patients with recurrent PC are insufficient. ICIs, which are considered a milestone in oncology, may provide hope for the future therapy of this rare cancer.

\section{AUTHOR CONTRIBUTIONS}

GF, SDM, AD, TFl, and TFe were responsible for the design, the methodology, the draft preparation, the reviewing, and editing. $\mathrm{AC}$ and AF were responsible for the supervision. All authors contributed to the article and approved the submitted version.

\section{FUNDING}

This work was supported by the Italian Ministry of Education, University and Research (MIUR): PRIN 2017Z3N3YC, and by University of Sassari, Italy: Research Funding 2019-2020 to GF. 


\section{ACKNOWLEDGMENTS}

This paper is part of the 'Neuroendocrine Tumors Innovation Knowledge and Education' project led by Annamaria Colao and Antongiulio Faggiano, which aims at increasing the knowledge on NET. We would like to acknowledge all the Collaborators of the "NIKE" project: Manuela Albertelli-Genova; Barbara Altieri-Wurzburg; Luigi Barrea-Napoli; Filomena BottiglieriNapoli; Severo Campione-Napoli; Federica de Cicco-Napoli; Alessandra Dicitore-Milano; Diego Ferone-Genova; Francesco Ferraù-Messina; Erika Grossrubatscher-Milano; Marco Gallo-

\section{REFERENCES}

1. Lenschow C, Fuss CT, Kircher S, Buck A, Kickuth R, Reibetanz J, et al. Case Report: Abdominal Lymph Node Metastases of Parathyroid Carcinoma: Diagnostic Workup, Molecular Diagnosis, and Clinical Management. Front Endocrinol (Lausanne) (2021) 12:643328. doi: 10.3389/ fendo.2021.643328

2. Cetani F, Pardi E, Marcocci C. Parathyroid Carcinoma. Front Horm Res (2019) 51:63-76. doi: 10.1159/000491039

3. Bollerslev J, Schalin-Jäntti C, Rejnmark L, Siggelkow H, Morreau H, Thakker R, et al. Unmet Therapeutic, Educational and Scientific Needs in Parathyroid Disorders: Consensus Statement From the First European Society of Endocrinology Workshop (PARAT). Eur J Endocrinol (2019) 181(3):P1P19. doi: 10.1530/EJE-19-0316

4. Franzin R, Netti GS, Spadaccino F, Porta C, Gesualdo L, Stallone G, et al. The Use of Immune Checkpoint Inhibitors in Oncology and the Occurrence of AKI: Where do We Stand? Front Immunol (2020) 11:574271. doi: 10.3389/ fimmu.2020.574271

5. Robert C. A Decade of Immune-Checkpoint Inhibitors in Cancer Therapy. Nat Commun (2020) 11. doi: 10.1038/s41467-020-17670-y

6. Park D, Airi R, Sherman M. Microsatellite Instability Driven Metastatic Parathyroid Carcinoma Managed With the Anti-PD1 Immunotherapy, Pembrolizumab. BMJ Case Rep (2020) 13(9). doi: 10.1136/bcr-2020-235293

7. Eisenhauer EA, Therasse P, Bogaerts J, Schwartz LH, Sargent D, Ford R, et al. New Response Evaluation Criteria in Solid Tumours: Revised RECIST Guideline (Version 1.1). Eur J Cancer (2009) 45(2):228-47. doi: 10.1016/ j.ejca.2008.10.026

8. Topalian SL, Hodi FS, Brahmer JR, Gettinger SN, Smith DC, McDermott DF, et al. Safety, Activity, and Immune Correlates of Anti-PD-1 Antibody in Cancer. N Engl J Med (2012) 366(26):2443-54. doi: 10.1056/NEJMoa1200690

9. Reck M, Rodríguez-Abreu D, Robinson AG, Hui R, Csőszi T, Fülöp A, et al. KEYNOTE-024 Investigators. Pembrolizumab Versus Chemotherapy for PDL1-Positive Non-Small-Cell Lung Cancer. N Engl J Med (2016) 375(19):182333. doi: 10.1056/NEJMoa1606774

10. Du X, Wang L, Shen B, He H, Chang H, Wei B. Clinical Significance of Pd-L1 Expression in Parathyroid Cancer. Acta Endocrinol (Buchar) (2016) 12 (4):383-6. doi: 10.4183/aeb.2016.383

11. Larkin J, Chiarion-Sileni V, Gonzalez R, Grob JJ, Cowey CL, Lao CD, et al. Combined Nivolumab and Ipilimumab or Monotherapy in Untreated Melanoma. N Engl J Med (2015) 373(1):23-34. doi: 10.1056/ NEJMoa1504030

12. Wolchok JD, Chiarion-Sileni V, Gonzalez R, Rutkowski P, Grob J-J, Cowey CL, et al. Overall Survival With Combined Nivolumab and Ipilimumab in Advanced Melanoma. N Engl J Med (2017) 377(14):1345-56. doi: 10.1056/ NEJMoa1709684

13. Bongiovanni M, Rebecchini C, Saglietti C, Bulliard J-L, Marino L, de Leval L, et al. Very Low Expression of PD-L1 in Medullary Thyroid Carcinoma. Endocr Relat Cancer (2017) 24(6):L35-8. doi: 10.1530/ERC-17-0104

14. Samstein RM, Lee C-H, Shoushtari AN, Hellmann MD, Shen R, Janjigian YY, et al. Tumor Mutational Load Predicts Survival After Immunotherapy Across Multiple Cancer Types. Nat Genet (2019) 51(2):202-6. doi: 10.1038/s41588018-0312-8
Torino; Elisa Giannetta-Roma; Federica Grillo-Genova; Elia Guadagno-Napoli; Valentina Guarnotta-Palermo; Andrea M. Isidori-Roma; Andrea Lania-Milano; Andrea Lenzi-Roma; Fabio Lo Calzo-Avellino; Pasquale Malandrino-Catania; Erika Messina-Messina; Roberta Modica-Napoli; Giovanna Muscogiuri-Napoli; Genoveffa Pizza-Avellino; Riccardo PofiRoma; Giulia Puliani-Roma; Carmen Rainone-Napoli; Paola Razzore-Torino; Laura Rizza-Roma; Manila Rubino -Milano; Rosa Maria Ruggieri-Messina; Emilia Sbardella-Roma; Franz Sesti-Roma; Mary Anna Venneri-Roma; Giovanni VitaleMilano; Maria Chiara Zatelli-Ferrara.

15. Yang RK, Qing Y, Jelloul FZ, Routbort MJ, Wang P, Shaw K, et al. Identification of Biomarkers of Immune Checkpoint Blockade Efficacy in Recurrent or Refractory Solid Tumor Malignancies. Oncotarget (2020) 11 (6):600-18. doi: 10.18632/oncotarget.27466

16. Kang H, Pettinga D, Schubert AD, Ladenson PW, Ball DW, Chung JH, et al. Genomic Profiling of Parathyroid Carcinoma Reveals Genomic Alterations Suggesting Benefit From Therapy. Oncologist (2019) 24(6):791-7. doi: 10.1634/theoncologist.2018-0334

17. Schmidt C. The Benefits of Immunotherapy Combinations. Nature (2017) 552 (7685):S67-9. doi: 10.1038/d41586-017-08702-7

18. Ma X, Zhang Y, Wang S, Wei H, Yu J. Immune Checkpoint Inhibitor (ICI) Combination Therapy Compared to Monotherapy in Advanced Solid Cancer: A Systematic Review. J Cancer (2021) 12(5):1318-33. doi: $10.7150 /$ jca.49174

19. Dai C, Liang S, Sun B, Kang J. The Progress of Immunotherapy in Refractory Pituitary Adenomas and Pituitary Carcinomas. Front Endocrinol (2020) 11. doi: $10.3389 /$ fendo.2020.608422

20. Fanciulli G, Di Molfetta S, Dotto A, Florio T, Feola T, Rubino M, et al. Emerging Therapies in Pheochromocytoma and Paraganglioma: Immune Checkpoint Inhibitors in the Starting Blocks. J Clin Med (2020) 10(1). doi: $10.3390 /$ jcm10010088. null.

21. Karwacka I, Obołończyk Ł, Kaniuka-Jakubowska S, Sworczak K. The Role of Immunotherapy in the Treatment of Adrenocortical Carcinoma. Biomedicines (2021) 9(2). doi: 10.3390/biomedicines9020098

22. Di Molfetta S, Dotto A, Fanciulli G, Florio T, Feola T, Colao A, et al. Immune Checkpoint Inhibitors: New Weapons Against Medullary Thyroid Cancer? Front Endocrinol (2021) 12:667784. doi: 10.3389/fendo. 2021.667784

23. Manohar S, Kompotiatis P, Thongprayoon C, Cheungpasitporn W, Herrmann J, Herrmann SM. Programmed Cell Death Protein 1 Inhibitor Treatment is Associated With Acute Kidney Injury and Hypocalcemia: Meta-Analysis. Nephrol Dial Transplant (2019) 34(1):108-17. doi: 10.1093/ ndt/gfy105

24. Nalluru SS, Piranavan P, Ning Y, Ackula H, Siddiqui AD, Trivedi N. Hypocalcemia With Immune Checkpoint Inhibitors: The Disparity Among Various Reports. Int J Endocrinol (2020) 2020:7459268. doi: 10.1155/2020/ 7459268

Conflict of Interest: The authors declare that the research was conducted in the absence of any commercial or financial relationships that could be construed as a potential conflict of interest.

The reviewer MG declared a past co-authorship with several of the authors TFe, $\mathrm{AC}$, and $\mathrm{AF}$ to the handling editor.

Copyright (C) 2021 Fanciulli, Di Molfetta, Dotto, Florio, Feola, Colao, Faggiano and NIKE Group. This is an open-access article distributed under the terms of the Creative Commons Attribution License (CC BY). The use, distribution or reproduction in other forums is permitted, provided the original author(s) and the copyright owner(s) are credited and that the original publication in this journal is cited, in accordance with accepted academic practice. No use, distribution or reproduction is permitted which does not comply with these terms. 